\title{
Wie genau ist die computergestützte Melanomdiagnostik?
}

Unzureichende Angebote an professionellen Hautkrebs-Screenings könnten durch automatische Bilddiagnose-Methoden ausgeglichen werden. Doch bis diese Anwendung in der Praxis finden, sind noch einige Fallstricke zu beheben, so die Ergebnisse einer aktuellen Metaanalyse.

In einer Metaanalyse [1] befassten sich Vincent Dick und sein Team der Dermatologic Imaging Forschungsgruppe der Medizinischen Universität in Wien mit der Messgenauigkeit von computergestützten Diagnoseverfahren des malignen Melanoms. 70 Studien konnten die Forschenden letztendlich in ihrer Metaanalyse auswerten. Sie ergaben für die automatische Melanomdiagnostik eine Sensitivität von $74 \%$ und eine Spezifität von $84 \%$. Verglichen mit der Einschätzung von Experten zeigte die computergestützte Diagnostik eine ähnliche Sensitivität, jedoch eine um $10 \%$ geringere Spezifität. (• Abb. 1) Der Unterschied ist statistisch jedoch nicht signifikant, und nur 14 der 70 Studien lieferten Daten zu dieser Fragestellung.

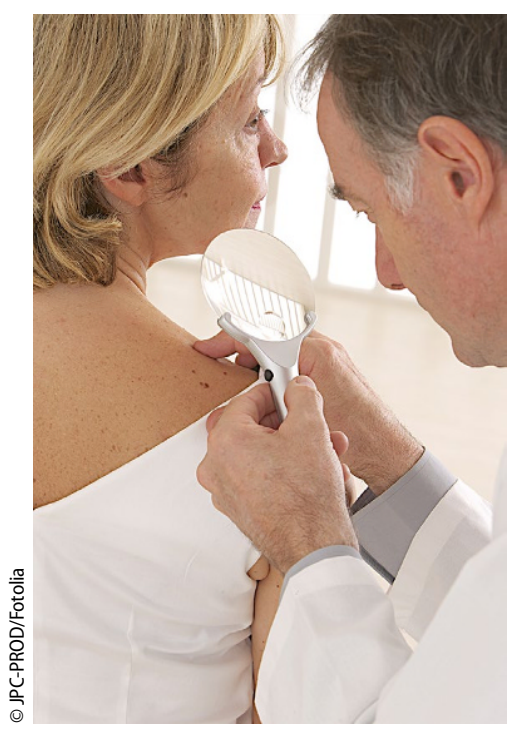

Abb. $1 \Delta$ Verglichen mit der Einschätzung von Experten zeigte die computergestützte Diagnostik eine ähnliche Sensitivität, jedoch eine um $10 \%$ geringere Spezifität
Je nach Studiencharakteristika zeigten sich unterschiedlich ausgeprägte Ergebnisse. Deutlich mehr falsch negative Diagnosen wurden in Studien mit unabhängigen Stichproben gestellt. Und auch die Methode der Computerdiagnostik bestimmte die Genauigkeit der Diagnosestellung: Deep-Learning-Anwendungen zeigten eine signifikant um mindesten $15 \%$ Prozentpunkte höhere Spezifität als die beiden anderen in der Metaanalyse erfassten Methoden (Computer Vision und Hardware-basierte Methoden).

\section{Übertragbarkeit auf Realität wird angezweifelt}

Die Heterogenität der Daten dieser Metaanalyse erschwert eine Verallgemeinerung und Übertragung auf die Praxis, und das Risiko der Verzerrung ist hoch. In den zu knapp $80 \%$ aus dem Feld der Computerwissenschaften stammenden Studien wurden weiterhin typische medizinische Problemstellungen des Melanom-Screenings nicht betrachtet, wie die Mehrdeutigkeit von pathologischen Berichten oder der Umgang mit Unsicherheit in der Praxis.

Oft findet in Maschinen-Lern-Experimenten außerdem eine Überanpassung statt, besonders wenn die für das Training herangezogenen Daten stark von einer klinischen Realsituation abweichen. Seltene Erkrankungen der Haut könnten in einer tatsächlichen Screening-Situation, wenn nicht ausreichend im Trainingslauf vorhanden, fälschlicherweise als Melanom identifiziert werden.

Um die Möglichkeiten von computerbasierten Diagnostiksystemen ein-

\section{Das Wichtigste in Kürze}

Wie verhalten sich Sensitivität und Spezifität bei computergestützten Diagnoseverfahren des Melanoms? Insgesamt diagnostizierten die Systeme mit einer Sensitivität von $74 \%$ und einer Spezifität von $84 \%$ Melanome. Differenziert nach Studiencharakteristika variierten diese Werte teils stark. Bedeutung: Computergestützte Screeningsysteme scheinen akkurate Diagnosen zu stellen, weitere Studien sind jedoch nötig, um deren Potential für die Praxis abschätzen zu können Einschränkung: Heterogene Charakteristika der ausgewerteten Studien, knapp $80 \%$ der Studien sind ohne medizinische Fokussierung verfasst

deutig einschätzen zu können, wären Studien nötig, die unter Realbedingungen den Einsatz solcher Anwendungen testen. Ohne eine klare Vorgabe des Settings und der Aufgabe, seien ein gezieltes Training sowie ein aussagekräftiger Test nicht möglich und die Technik sei am Ende für die Praxis nutzlos, schlussfolgern die Autoren.

\section{Literatur}

1. Dick V et al (2019) Accuracy of ComputerAided Diagnosis of Melanoma A Meta-analysis. JAMA Dermatol. https:// doi.org/10.1001/jamadermatol.2019.1375

hautnah $2019 \cdot 18: 133$

https://doi.org/10.1007/s12326-0190339-3

(C) Springer-Verlag GmbH Austria, ein Teil von Springer Nature 2019
Quelle: SpringerMedizin.de, Anika Aßfalg 